\title{
Faster clean catch urine collection (Quick-Wee method) from infants: randomised controlled trial
}

\author{
Jonathan Kaufman, 1,2,3 Patrick Fitzpatrick,,2 Shidan Tosif,,2,3 Sandy M Hopper, 1,2 Susan M Donath,2,3 \\ Penelope A Bryant, ${ }^{1,2,3}$ Franz E Babl1,2,3
}

${ }^{1}$ Royal Children's Hospital, Parkville, Victoria, Australia

${ }^{2}$ Murdoch Children's Research Institute, Parkville, Victoria, Australia

${ }^{3}$ Department of Paediatrics, Faculty of Medicine Dentistry and Health Sciences, University of Melbourne, Parkville, Victoria, Australia

Correspondence to: FE Babl Murdoch Children's Research Institute, Parkville, Victoria 3052, Australia

franz.babl@rch.org.au

Cite this as: $B M$ I 2017:357::1341 http://dx.doi.org/10.1136/bmj.j1341 Accepted: 10 March 2017

\begin{abstract}
OBJECTIVE

To determine if a simple stimulation method increases the rate of infant voiding for clean catch urine within five minutes.

DESIGN

Randomised controlled trial.

SETTING

Emergency department of a tertiary paediatric hospital, Australia.

PARTICIPANTS

354 infants (aged 1-12 months) requiring urine sample collection as determined by the treating clinician. 10 infants were subsequently excluded.

INTERVENTIONS

Infants were randomised to either gentle suprapubic cutaneous stimulation ( $\mathrm{n}=174$ ) using gauze soaked in cold fluid (the Quick-Wee method) or standard clean catch urine with no additional stimulation $(n=170)$, for five minutes.

\section{MAIN OUTCOME MEASURES}

The primary outcome was voiding of urine within five minutes. Secondary outcomes were successful collection of a urine sample, contamination rate, and parental and clinician satisfaction with the method.

RESULTS

The Quick-Wee method resulted in a significantly higher rate of voiding within five minutes compared with standard clean catch urine $31 \% \mathrm{~V}$ $12 \%, \mathrm{P}<0.001)$, difference in proportions $19 \%$ favouring Quick-Wee (95\% confidence interval for difference $11 \%$ to $28 \%$ ). Quick-Wee had a higher rate of successful urine sample collection $30 \% \mathrm{~V}$ $9 \%, \mathrm{P}<0.001)$ and greater parental and clinician satisfaction (median $2 v 3$ on a 5 point Likert
\end{abstract}

\section{WHAT IS ALREADY KNOWN ON THIS TOPIC}

Urinary tract infection (UTI) is a common early childhood febrile illness, and a urine sample is required for diagnosis or exclusion

Urine sample collection can be challenging in pre-continent children, and current collection methods have limitations

The clean catch urine collection method is recommended by UK NICE guidelines, but can be time consuming or unsuccessful

\section{WHAT THIS STUDY ADDS}

A simple suprapubic cutaneous stimulation method using gauze soaked in cold fluid to trigger voiding (the "Quick-Wee" method) significantly increased the voiding and success rate of clean catch urine for infants aged 1-12 months

The Quick-Wee method requires minimal resources and is a simple way to trigger faster voiding for clean catch urine from infants in the acute care setting scale, $\mathrm{P}<0.001)$. The difference in contamination between Quick-Wee and standard clean catch urine was not significant $(27 \%$ v $45 \%, \mathrm{P}=0.29)$. The number needed to treat was 4.7 (95\% confidence interval 3.4 to 7.7 ) to successfully collect one additional urine sample within five minutes using Quick-Wee compared with standard clean catch urine.

\section{CONCLUSIONS}

Quick-Wee is a simple cutaneous stimulation method that significantly increases the five minute voiding and success rate of clean catch urine collection.

TRIAL REGISTRATION

Australian New Zealand Clinical Trials Registry

ACTRN12615000754549.

\section{Introduction}

Urinary tract infections (UTIs) are common in young children, affecting $5-7 \%$ of febrile children under 2 years of age. ${ }^{12}$ Investigation of potential UTIs should be considered in cases of unexplained fever in this age group, as clinical signs may be non-specific. ${ }^{34}$ The diagnosis of a UTI has important implications for follow-up, and delayed treatment can result in morbidity, including renal scarring ${ }^{56}$ and serious bacterial infection. ${ }^{7}$ Expedient collection of a urine sample allows for dipstick urinalysis and microscopy screening to guide immediate clinical decision making for treatment initiation, ${ }^{8}$ although a positive culture result with pyuria is the gold standard for definitive diagnosis. ${ }^{7}$ However, obtaining urine from pre-continent children can be difficult and time consuming. Despite urine samples being so commonly required in clinical practice, all current collection methods have limitations. Choosing the method of collection must balance reliability, speed, contamination, and invasiveness. Accordingly, clinical guidelines have differing recommendations, ${ }^{47}$ clinician preferences vary, ${ }^{9-11}$ and ongoing debate about the optimal collection method exists in the literature. ${ }^{1213}$

Non-invasive methods for urine collection may be regarded as practical and convenient and acceptable for children who do not require urgent antibiotic treatment. ${ }^{7}$ Urine collection bags are often used ${ }^{1114}$ but have unacceptably high false positive and contamination rates. ${ }^{1516}$ The culture results from a bag urine sample and antibiotic sensitivities therefore cannot be used reliably to direct treatment. Clean catch urine collection involves waiting for a nappy-free child to void spontaneously, and it is the recommended method of urine collection by the UK National Institute for Health and Care Excellence guidelines. ${ }^{4}$ Contamination rates of 5-27\% have been reported for clean catch urine, ${ }^{16-19}$ 
much lower than other non-invasive methods. ${ }^{151619}$ In practice though, clean catch urine collection can tie up valuable resources in clinical settings, with average waiting times of $30-71$ minutes, ${ }^{20} 21$ of which only $64 \%$ of first attempts are successful. ${ }^{21}$ In contrast to the UK, the American Academy of Pediatrics guidelines recommend non-invasive samples can be used for screening; but an invasive catheterisation or suprapubic aspiration sample is required for definitive diagnosis and before starting antibiotic treatment. ${ }^{7}$ Invasive samples have lower contamination rates ${ }^{16}$ but cause pain and distress for children and require equipment and technical expertise to collect. For these reasons invasive methods may not be favoured by some clinicians, ${ }^{9-11}$ and might be impractical outside hospital settings. ${ }^{13}$

These difficulties with urine sample collection can lead to misdiagnosis or a missed diagnosis of UTI. One study found that $32 \%$ of children aged less than 2 years had no urinalysis or culture performed for outpatient UTI episodes treated with antibiotics. ${ }^{22}$ An ideal method of urine collection would be non-invasive, fast, gentle, and simple, and such a method has been identified as a priority for future research. ${ }^{72324}$ Anecdotally, clinicians may have noted that some children void spontaneously during the routine cleaning of the perigenital area before urine collection. This cold, wet stimulation likely triggers cutaneous voiding reflexes, which remain under-investigated as a mechanism for improved urine collection. In animal studies perigenital-bladder spinal reflexes can be stimulated mechanically or when mothers lick the perigenital skin of their newborns. ${ }^{2526}$ In humans, suprapubic stimulation is proposed to trigger parasympathetic detrusor contraction through the exteroceptive somato-bladder reflex mechanism. ${ }^{27}$ We hypothesised that gentle suprapubic cutaneous stimulation using gauze soaked in cold fluid (the Quick-Wee method) might hasten bladder voiding for clean catch urine by triggering early childhood cutaneous voiding reflexes. We evaluated the efficacy of this new voiding stimulation method to increase the rate of urine voiding within five minutes for infants in the emergency department.

\section{Methods}

The full study protocol is published and available in an open access journal. ${ }^{28}$

\section{Trial design, participants, and setting}

This was a randomised, prospective, non-blinded superiority trial, conducted from September 2015 to April 2016 in a single tertiary paediatric emergency department at the Royal Children's Hospital, Melbourne Australia. The emergency department has 85000 presentations annually.

Eligible participants were pre-continent infants aged 1-12 months (corrected for prematurity if $<36$ weeks' gestation) where a urine sample was required and the treating clinician determined clean catch urine to be an appropriate method of collection. Neonates (aged $<1$ month) were not included as our institution does not recommend non-invasive urine collection to investigate
UTI in this age group because of their greater risk of serious bacterial infection. We excluded infants with anatomical or neurological abnormalities affecting voiding or sensation.

No changes to methods were made after the trial commenced. The study is reported in accordance with the Consolidated Standards for Reporting Trials (CONSORT) statement for randomised trials. ${ }^{29}$

Clinicians in the emergency department (nursing and medical) were trained to recruit participants and implement the intervention with face-to-face departmental education sessions and written instructions. Infants suitable for inclusion were identified by clinicians, who made decisions about the appropriate method of urine sample collection on clinical grounds. Suitable participants were recruited consecutively.

We assigned participants randomly, in a 1:1 ratio, to receive either the intervention (Quick-Wee) or usual care (standard clean catch urine with no additional stimulation) for up to five minutes. An independent statistician prepared the randomisation schedule using random permuted blocks with at least three different block sizes to ensure concealment of allocation. Opaque envelopes concealing the allocation, within sealed individual study packs, were used to assign the intervention in the emergency department. Study packs were kept available from a locked study box from which they could only be taken sequentially. Owing to the obvious nature of the intervention, blinding was not possible after randomisation and assignment to intervention.

\section{Interventions}

The clinician provided a written study information handout, verbally explained the procedure to the parent/carer, and obtained verbal consent.

The clinician prepared the child for the clean catch urine attempt, opened the opaque randomisation envelope by the bedside, then immediately removed the nappy and started the timer. A parent, carer, or clinician was ready to catch a urine sample if the child voided, and the genital orifice was cleaned for $10 \mathrm{sec}-$ onds with sterile water at room temperature (standard practice). In the usual care arm the clinician waited for the child to void spontaneously, until clean catch urine was obtained or the timer reached five minutes. In the intervention arm the clinician (or parent/carer with supervision) additionally rubbed the suprapubic area of the child in a circular pattern with gauze soaked in cold saline held with disposable plastic forceps (fig 1), until clean catch urine sample was obtained or the timer reached five minutes. One operator performed the intervention.

The timer was stopped if the child voided and clean catch urine was obtained, and time to void was recorded. Alternatively a missed catch, failure to void at five minutes, or reason for abandoning the procedure was recorded, as well as parental and clinician satisfaction with the method. Children who were randomised and voided during the 10 second cleaning phase (which was part of the total five minute study period) were included in the intention-to-treat analysis. 


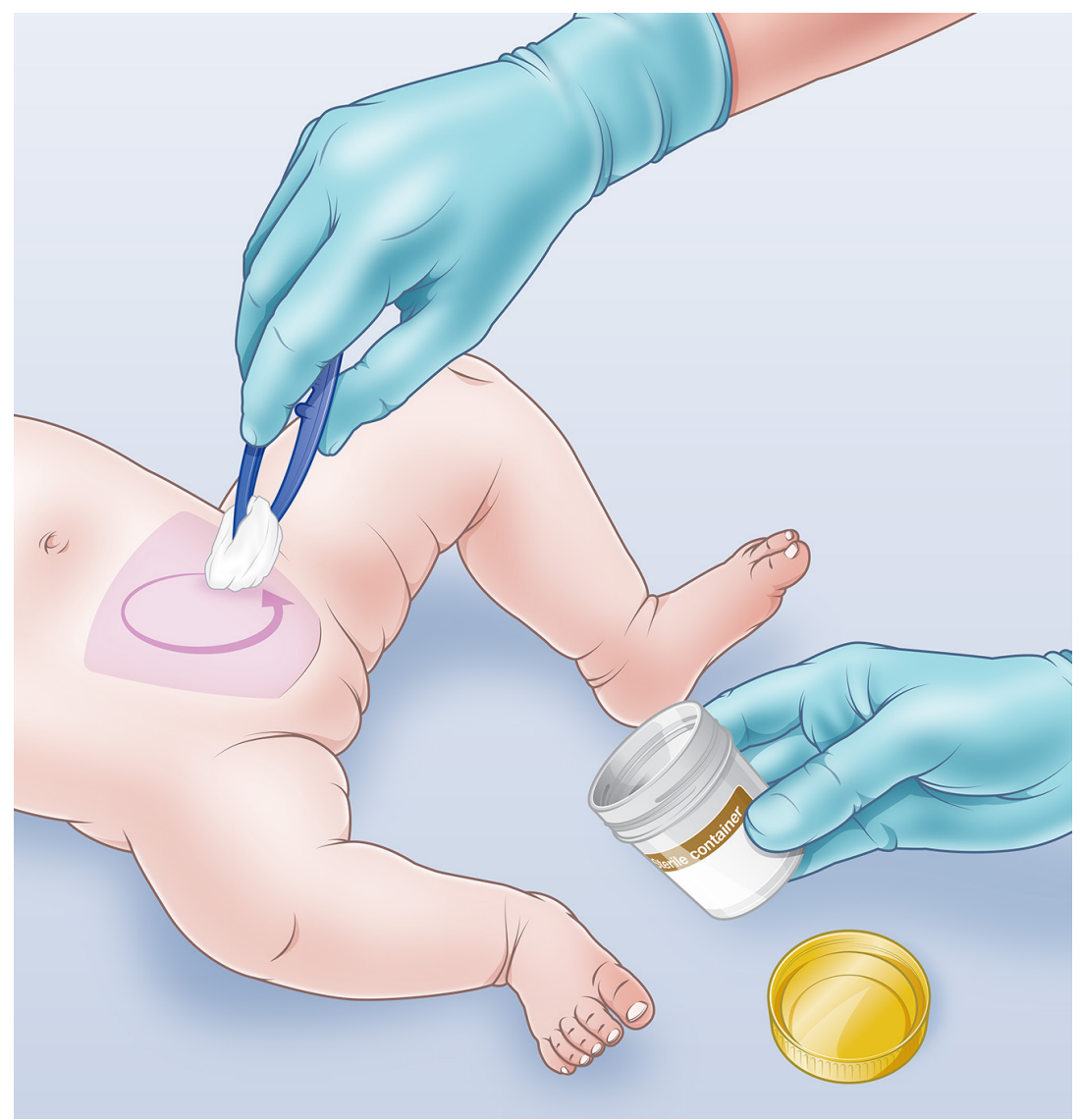

Fig 1 | Quick-Wee voiding stimulation method of gentle cutaneous suprapubic stimulation using gauze soaked in cold fluid. Image courtesy of Bill Reid, Royal Children's Hospital creative studio
Secondary outcome measures were if the child voided, whether any urine was successfully caught in the specimen jar or was missed (binary yes or no outcome: clean catch urine collected if voided), contamination of clean catch urine samples obtained within five minutes (binary yes or no outcome: urine culture contamination as per hospital laboratory definition, $\geq 3$ colony types with colony forming units (CFU) $\geq 10^{6} / \mathrm{L}$, or $\geq 2$ colony types with $\mathrm{CFU} \geq 10^{7} / \mathrm{L}$ where the predominant organism was a contaminant), and parental and clinician satisfaction with the urine collection method (5 point Likert satisfaction rating scale).

\section{Data collection}

Clinicians in the emergency department recorded data on paper case record forms. The data were then entered by the research team into the REDCap (Research Electronic Data Capture) electronic database. ${ }^{32}$

Demographic and participant data recorded included age, sex, relevant medical comorbidities, previous UTI, clinical indication for urine collection as recorded by the clinician, and reasons for exclusions and refusals.

Clinical data recorded included whether the child voided within five minutes, and time taken to void; successful catch of urine sample if voided; person performing the standardised cleaning (parent, doctor, or nurse); person performing the suprapubic stimulation in the intervention group (parent, doctor, or nurse); person catching the urine (parent, doctor, or nurse); adverse events; whether the urine collection attempt was abandoned before five minutes and, if so, reason for abandonment; and parental and clinician satisfaction with the urine collection method.

Clinical data collected subsequently by the research team (by linking with laboratory data and hospital records) included positive and contaminated urine culture results, admission to hospital, and start of antibiotic treatment.

\section{Sample size calculation}

A sample size of 354 participants (177 in each group) was based on power calculations using data from planned preceding baseline and pilot studies. An initial prospective observational baseline study determined the success of standard clean catch urine in young pre-continent children, and in preliminary data children aged $<12$ months 21\% (12/57) voided within five minutes. ${ }^{33}$ We surveyed a panel of 20 expert clinicians (paediatric emergency doctors and paediatricians at consultant level) who reported an increase in success rate of approximately $15 \%$ compared with standard clean catch urine collection would suggest that a simple technique such as Quick-Wee should be incorporated into their clinical practice. A pilot study of the new method showed that Quick-Wee was a feasible and acceptable intervention to obtain clean catch urine within five minutes. ${ }^{30}$ Thirty five per cent (7/20) of children voided using cold temperature saline for stimulation, suggesting that a difference of approximately $15 \%$ was a reasonable estimate of the treatment effect. Most successful voids were from infants

\section{Outcomes}

The primary outcome measure was voiding of urine within five minutes (binary yes or no outcome: voided). 
aged 1-12 months, an age group that was therefore chosen for the definitive trial.

A sample size of 322 participants (161 in each group) was required to have $80 \%$ power to detect a difference between groups of $21 \%$ (non-intervention arm) versus $35 \%$ (intervention arm) for voiding within five minutes. Power and sample size calculations were completed using an estimated total sample size for a two sample proportions test (Pearson's $\chi^{2}$ test). An additional 10\% of participants (16 in each group) were included to account for a small loss to follow-up of primary outcome results. Analysis was by intention to treat, consistent with CONSORT guidelines. ${ }^{2934}$

\section{Statistical analysis}

For the primary outcome we report rates of voiding urine within five minutes for each group with percentages, the absolute difference for percentage voiding between groups, and 95\% confidence intervals for each, and we calculate $P$ values using a $\chi^{2}$ test. We considered a $\mathrm{P}$ value $<0.05$ to be significant. For secondary outcomes we report the rates of successful urine collection and contamination for each group with percentages and 95\% confidence intervals, and median and interquartile ranges for parental and clinician satisfaction. The appropriate difference between groups was reported (difference of proportions for categorical outcomes, difference of medians for skewed continuous outcomes), together with the $95 \%$ confidence interval for the difference. We estimated $\mathrm{P}$ values using $\chi^{2}$ for categorical variables and $t$ test or Wilcoxon rank-sum tests for continuous variables.

In addition to the unadjusted analysis, all treatment comparisons for primary and secondary outcomes were

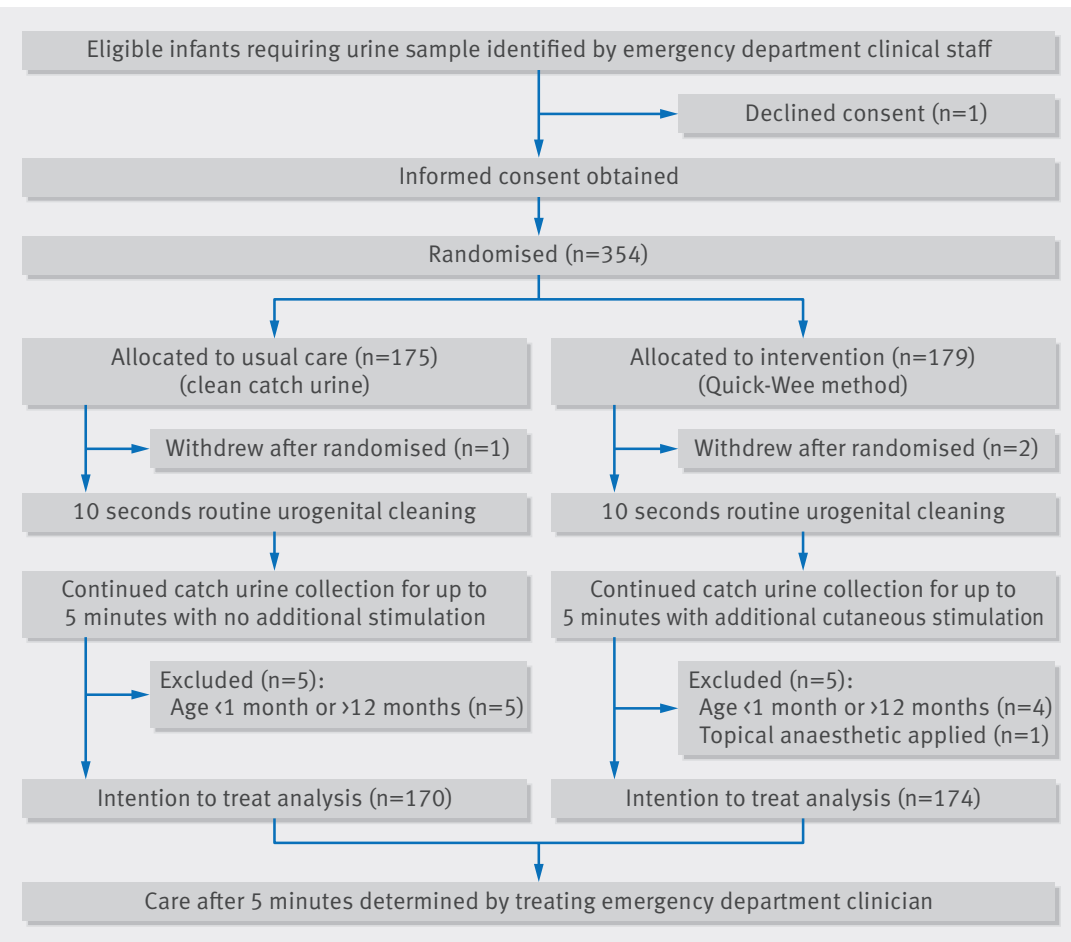

Fig 2 | Flow of participants through study adjusted for age and sex to account for any chance imbalance between the treatment groups with respect to these potentially confounding factors, using quantile and logistic regression models for continuous and binary outcomes, respectively. Statistical calculations and analyses were completed using Stata 14 (Stata Corp, TX). No interim analysis was performed.

\section{Patient involvement}

Feedback from the parents and carers of 40 participants in the preceding pilot study contributed to refinement and confirmation of the study research question and outcome measures. Parents and carers were asked to rate their satisfaction with the intervention and could provide additional comments. Parental satisfaction with the intervention in the pilot study was high and no respondents were dissatisfied with the intervention, ${ }^{30}$ demonstrating feasibility for this larger definitive trial.

Patients were not further involved in the design, recruitment, and conduct of the study. The burden of intervention was assessed with a parental satisfaction rating using a 5 point Likert scale, which was a secondary outcome measure of this study. Results of this study will be available to the wider community, including study participants, through publication in an open access journal.

\section{Results}

Overall, 354 participants were recruited and 10 were excluded (fig 2): five were aged less than 1 month, four were aged more than 12 months, and one had topical anaesthetic cream applied for a suprapubic aspiration procedure that affected sensation. One family declined consent to randomisation, and three families withdrew consent and requested sample collection by another method (one in the control group and two in the intervention group). No adverse events occurred during the trial, and no participants had missing primary outcome data. Two infants ( $0.6 \%$ of participants) in the intervention group voided during the 10 second cleaning procedure and were included in the intention-to-treat analysis.

Of 344 participants included in the analysis, 172 $(50 \%)$ were male with a mean age of 5.4 months (table 1). Clinical indications (could be $\geq 1$ ) for urine collection were predominantly fever of unknown origin $(n=144$, $42 \%)$, unsettled baby ( $n=132,38 \%)$, poor feeding $(n=76$, $22 \%)$, specifically suspected UTI ( $\mathrm{n}=60,17 \%)$, and vomiting $(\mathrm{n}=27,8 \%)$. Thirty three infants $(10 \%)$ had medical comorbidities (table 1). A UTI was present in eight of 55 infants (14\%) with laboratory culture results available, defined as growth of a uropathogenic organism with $\mathrm{CFU} \geq 10^{8} / \mathrm{L}$.

A total of 127 clinicians recruited one or more participants for the trial ( $\mathrm{n}=60$ (47\%) nursing, $\mathrm{n}=67$ (53\%) medical), and subsequently allocated roles for the procedure. Clinicians primarily performed the routine urogenital cleaning of the 354 infants in the trial ( $\mathrm{n}=152$ (43\%) nursing, $\mathrm{n}=174$ (49\%) medical, $\mathrm{n}=28$ (8\%) parent) and the Quick-Wee method for the 174 infants 


\begin{tabular}{|c|c|c|}
\hline Characteristics & $\begin{array}{l}\text { Quick-Wee } \\
(n=174)\end{array}$ & $\begin{array}{l}\text { Standard clean catch } \\
\text { urine }(n=170)\end{array}$ \\
\hline Male & $95(55)$ & $77(45)$ \\
\hline Mean (SD) age in months & $5.4(3.2)$ & $5.4(3.0)$ \\
\hline Previous UTI & $10(6)$ & $15(9)$ \\
\hline Antibiotics in past 24 hours & $9(5)$ & $10(6)$ \\
\hline \multicolumn{3}{|l|}{ Comorbidities: } \\
\hline Minor renal anomaly & $8(5)$ & $7(4)$ \\
\hline Congenital cardiac & $3(2)$ & $1(0.6)$ \\
\hline Seizure disorder & $3(2)$ & $0(0)$ \\
\hline Genetic syndrome & $0(0)$ & $2(1)$ \\
\hline Gastro-oesophageal reflux & $2(1)$ & $2(1)$ \\
\hline Other & $3(2)$ & $2(1)$ \\
\hline \multicolumn{3}{|l|}{ Indication for urine sample*: } \\
\hline Fever of unknown origin & $78(45)$ & $66(39)$ \\
\hline Unsettled baby & $66(38)$ & $66(39)$ \\
\hline Poor feeding & $33(19)$ & $43(25)$ \\
\hline Specifically suspected UTI & $29(17)$ & $27(16)$ \\
\hline Vomiting & $17(10)$ & $10(6)$ \\
\hline Failure to thrive & $1(1)$ & $7(4)$ \\
\hline Metabolic/electrolyte/jaundice & $0(0)$ & $3(2)$ \\
\hline Haematuria & $2(1)$ & $1(1)$ \\
\hline Other & $6(3)$ & $6(4)$ \\
\hline \multicolumn{3}{|l|}{ Hydration state: } \\
\hline Normal & $120(69)$ & $132(78)$ \\
\hline Mild & $48(28)$ & $33(19)$ \\
\hline Moderate & $5(3)$ & $2(1)$ \\
\hline Severe & $0(0)$ & $1(1)$ \\
\hline
\end{tabular}

\section{UTI=urinary tract infection.}

Apparent discrepancies are due to rounding.

*More than one indication may be selected.

in the intervention group ( $\mathrm{n}=89$ (51\%) nursing, $\mathrm{n}=82$ (47\%) medical, $n=3$ (2\%) parent). Parents primarily collected the urine of the 74 infants who voided within the five minute trial period $(\mathrm{n}=13(18 \%)$ nursing, $\mathrm{n}=8$ (11\%) medical, $n=46$ (62\%) parent, $n=7$ (9\%) not recorded).

Compared with standard clean catch urine, QuickWee had a higher rate of voiding within five minutes (table 2). In total, 54 out of 174 infants (31\%) voided within five minutes in the Quick-Wee group, compared with 20 out of $170(12 \%)$ in the standard clean catch urine group. The difference in proportions for the primary outcome was 19\% favouring Quick-Wee (95\% confidence interval for difference $11 \%$ to $28 \%$ ), with a risk ratio of 2.6.

Compared with standard clean catch urine, QuickWee had a similarly higher rate for successfully catching a urine sample and also higher parental and clinician satisfaction with the method of urine sample collection (table 2). The rate of contamination between groups was not statistically significant, with lower numbers for this outcome because not all participants had a urine sample processed for culture (table 2). The number needed to treat to successfully collect one additional urine sample within five minutes using Quick-Wee compared with standard clean catch urine was 4.7 (95\% confidence interval 3.4 to 7.7 ).

After adjustment for age and sex the primary outcome results were unchanged (data not shown). Risk difference and confidence intervals for successfully catching urine and for contamination, and difference in medians for parental and clinician satisfaction with the method, were also unchanged when adjusted for age and sex.

\section{Discussion}

Gentle suprapubic cutaneous stimulation with gauze soaked in cold fluid (the Quick-Wee method) led to a clinically and statistically significant increase in voiding and successful urine collection within five minutes for infants aged 1-12 months. The difference in contamination was not significant as confidence intervals overlapped, potentially due to lower numbers of available culture results. Importantly, parents and clinicians preferred the Quick-Wee method, reporting higher satisfaction than with standard clean catch urine.

\section{Strengths and limitations of this study}

To our knowledge this is the first randomised trial to demonstrate the efficacy of a voiding stimulation method for infants. Strengths of this study include the preceding baseline and pilot feasibility studies, its large sample size, and randomised study design. More than 100 clinicians in the emergency department rather than dedicated research staff performed the intervention, and all eligible infants were included regardless of feeding and hydration state, minimising

\begin{tabular}{|c|c|c|c|c|c|c|}
\hline Outcomes & $\begin{array}{l}\text { Quick-Wee } \\
(n=174)\end{array}$ & $\%(95 \% \mathrm{Cl})$ & $\begin{array}{l}\text { Standard } \\
\text { clean catch } \\
\text { urine }(n=170)\end{array}$ & $\%(95 \% \mathrm{Cl})$ & $\begin{array}{l}\text { Difference in } \\
\text { proportions or } \\
\text { medians }(95 \% \mathrm{Cl})\end{array}$ & $P$ value \\
\hline \multicolumn{7}{|l|}{ Primary outcome: } \\
\hline Voided $<5$ mins & $54 / 174$ & $31(24$ to 39$)$ & $20 / 170$ & $12(7$ to 18$)$ & 19 (11 to 28$)$ & $<0.001^{\star}$ \\
\hline \multicolumn{7}{|l|}{ Secondary outcomes: } \\
\hline Voided and successful catch & $52 / 174$ & 30 (23 to 37$)$ & $15 / 170$ & $9(5$ to 14$)$ & 21 (13 to 29$)$ & $<0.001^{*}$ \\
\hline Contaminationt & $12 / 44$ & 27 (15 to 43$)$ & $5 / 11$ & $46(17$ to 77$)$ & $18(-14$ to 50$)$ & $0.29 \ddagger$ \\
\hline Median (interquartile range) parent satisfaction§ & $2(1-3)$ & & $3(2-3)$ & & $1(0.6$ to 1.4$)$ & $<0.0019$ \\
\hline Median (interquartile range) clinician satisfaction§ & $2(1-3)$ & & $3(2-3)$ & & $1(0.6$ to 1.4$)$ & $<0.0019$ \\
\hline
\end{tabular}

†Small proportion of samples in each group not sent for culture by treating clinician.

$\ddagger$ Fisher's exact test.

$\S 5$ point Likert scale: $1=$ very satisfied, $2=$ satisfied, $3=$ neutral, $4=$ unsatisfied, $5=$ very unsatisfied.

१Wilcoxon rank sum test. 
selection bias and ensuring the generalisability of results. The method is simple and does not require any specialised equipment so it can be utilised in low resource settings as a successful example of frugal innovation. ${ }^{35}$ This study has some limitations. Given the obvious nature of the method it would not be possible to blind clinicians to the intervention. Neonates and pre-continent children aged more than 12 months were not included in the study.

\section{Comparison to other studies}

Previous randomised studies have examined novel methods to obtain more rapid clean catch urine in pre-continent children. These include a vibrating bladder stimulator for infants and toddlers in the emergency department (not effective) ${ }^{20}$ and lumbar/bladder stimulation for neonates in the neonatal intensive care unit (effective but limited to neonatal age group and setting). ${ }^{18}$

Three decades ago Taylor et al tested the concept of triggering voiding using a finger tap method, although this study was limited by not recording the time taken to void in over half the infants in the study and by lacking a comparison group. ${ }^{36}$ The lumbar/bladder stimulation method subsequently described by Herreros et al has been shown to have a promising success rate of $78-86 \%$ for clean catch urine within five minutes in a neonatal intensive care setting. ${ }^{1831}$ However, participants in these studies had a mean age of 7 or less days, urine was predominantly collected for reasons other than investigation of UTI or fever, and infants with poor hydration and feeding were excluded. This may limit the generalisability of these findings to children in the primary or acute care setting, who are predominantly older and often unwell and poorly hydrated when a UTI is suspected. The lumbar/bladder stimulation method also requires infants to be held up suspended under the arms while stimulation manoeuvres are performed and catching a sample is attempted. This technique requires two or three staff members, ${ }^{182331}$ and may be less feasible in larger and heavier infants. ${ }^{37}$

The lumbar/bladder stimulation method has recently been investigated in three non-randomised trials for infants in the emergency department and was found to have a lower success rate in this population. Valleix et al obtained clean catch urine within five minutes in $27 \%$ of pre-continent children aged more than 1 month, with difficulties managing the children's position in $46 \%$ of the participants. ${ }^{37}$ Labrosse et al obtained clean catch urine within five minutes in $49 \%$ of younger infants aged less than 6 months. ${ }^{38}$ Tran et al used a different method of two three-minute stimulation attempts separated by a 30 minute interval of bag urine collection during which the infant was given water, formula milk, or breast milk. ${ }^{23}$ Although results are thus not directly comparable, the study found an overall success rate of $40.9 \%$ for 142 non-ambulant infants aged less than 2 years, which reduced with age, from $88.9 \%$ (newborn) to $28.6 \%$ (age $>1$ year). All three studies reported greater success in younger infants. This reduced potency in older infants may be expected given the hypothesis of stimulating newborn cutaneous voiding reflexes, which diminish with age. ${ }^{39}$

The contamination rate for the Quick-Wee method (27\%) is similar to that reported in previous voiding stimulation studies $\left(16 \%,{ }^{38} 24 \%,{ }^{18}\right.$ and $\left.38 \%{ }^{23}\right)$. Exact microbiological definitions of contamination, however, are not established in the literature and guidelines and vary between laboratories and institutions and thus may not be directly comparable. Clinical decisions about specimen collection and treatment in the presence of contamination should consider the clinical context, urinalysis, and the presence or absence of pyuria on microscopy.

Cold thermal stimulation has been suggested to pose a risk of cold burns to sensitive skin ${ }^{20}$; however, in the 174 infants in the intervention arm of the current study there were no such adverse events and there was high parental and clinician satisfaction associated with stimulation using gauze soaked in cold fluid refrigerated to a temperature of $2.8^{\circ} \mathrm{C}$.

\section{Unanswered questions and future research}

This trial used gauze soaked in cold fluid for stimulation over a period of five minutes. We used saline for the stimulation fluid so that ampoules could be easily identified in the trial, but any clean cold fluid could be used. Although our pilot study suggested that cold stimulus was more effective than stimulus at room temperature, ${ }^{30}$ future trials examining the temperature of stimulation applied to trigger voiding or a repeated application of the stimulation may further enhance the success of this method. Children in the neonatal age range were not included in the trial, but younger infants have been shown to be more responsive to stimulation of newborn cutaneous voiding reflexes. ${ }^{23} 3738$ This method could be further evaluated for neonatal patients where urine is being collected for reasons other than investigation of a UTI. We hypothesised that stimulating faster and more forceful voiding may reduce sample contamination, and although contamination was lower with the intervention this did not reach statistical significance. Contamination of clean catch urine may be related to urine flushing of the foreskin or vagina, which is seen on micturating cystourethrogram, 40 and higher contamination has been found from initial void rather than midstream samples. ${ }^{41}$ Future research to further reduce the contamination of clean catch urine by incidental skin flora, such as through standardised methods to clean the perigenital area, 42 may be of additional benefit.

\section{Conclusions and implications for clinicians and policy makers}

UTI is common in unwell young children presenting to primary and acute care, but reaching or excluding the diagnosis is not straightforward. ${ }^{2434}$ Collecting urine samples from young pre-continent children is challenging and the limitations of existing methods are well 
known. National Institute for Health and Care Excellence guidelines on UTI recommend clean catch urine collection, although in practice, attempts can be time consuming or unsuccessful. ${ }^{21}$ Voiding stimulation methods such as Quick-Wee can enhance clean catch urine collection by increasing the speed and success of obtaining urine. Timely collection of specimens is important and has many benefits, including expediting patient progress and clinical decision making in primary and acute care settings, reducing unnecessary or broad spectrum antibiotic treatment, ${ }^{22}$ preventing morbidity from UTI, and limiting unnecessary representation and follow-up to health services for missed diagnosis and misdiagnosis. Voiding stimulation methods could be easily incorporated into urine collection strategies in guidelines and clinical practice where non-invasive collection is indicated. Where appropriate this may spare some children the pain and distress associated with catheter and suprapubic aspiration procedures. The Quick-Wee method requires minimal resources and is a simple way to trigger faster voiding for clean catch urine from infants in the acute care setting.

We thank the families and clinical staff participating in this trial. The Murdoch Children's Research Institute and the Melbourne Children's Trial Centre assisted with the study design, data management, and analysis and interpretation of data for this study. Staff from the emergency department of the Royal Children's Hospital assisted with participant recruitment.

Contributors: PAB and FEB are joint senior authors. JK had the original idea for the study, and all authors contributed to the trial design and plan. JK and FEB were responsible for obtaining trial funding. JK, PF, ST, $\mathrm{SH}$, and $\mathrm{FB}$ were responsible for supervising the study and data acquisition in the emergency department. JK and SMD carried out the statistical analysis. JK drafted the manuscript, which was revised by all authors. JK and FEB are guarantors.

Funding: This study was funded by a philanthropic research project grant from the Shepherd Foundation. The foundation was independent of this trial and its researchers and had no part in the study design, data collection, data interpretation and analysis, writing of the manuscript, or decision to submit the manuscript for publication. JK has been part funded by an Avant doctor in training research scholarship and Royal Australasian College of Physicians Basser research entry scholarship. FEB is supported in part by a National Health and Medical Research Council Centre of Excellence research grant for paediatric emergency medicine (GNT 1058560) and a Royal Children's Hospital foundation grant, Melbourne Australia. The Royal Children's Hospital Melbourne receives infrastructure support from the Victorian government's infrastructure support programme, Melbourne, Australia.

Competing interests: All authors have completed the ICMJE uniform disclosure form at www.icmje.org/coi_disclosure.pdf and declare: the trial was supported by a philanthropic research grant from the Shepherd Foundation; no financial relationships with any organisations that might have an interest in the submitted work in the previous three years; no other relationships or activities that could appear to have influenced the submitted work.

Ethical approval: The study protocol (HREC reference 35083A) was approved by the Royal Children's Hospital human research ethics committee. All participants gave informed consent.

Data sharing: No additional data available.

Transparency: The manuscript's guarantors (JK and FEB) affirm that the manuscript is an honest, accurate and transparent account of the study being reported; that no important aspects of the study have been omitted; and that any discrepancies from the study as planned (and if relevant, registered) have been explained.

This is an Open Access article distributed in accordance with the Creative Commons Attribution Non Commercial (CC BY-NC 4.0) license, which permits others to distribute, remix, adapt, build upon this work non-commercially, and license their derivative works on different terms, provided the original work is properly cited and the use is non-

commercial. See: http://creativecommons.org/licenses/by-nc/4.0/.

1 Hoberman A, Wald ER. Urinary tract infections in young febrile children. Pediatr Infect Dis / 1997;16:11-7. doi:10.1097/00006454-199701000-00004.

2 Shaikh N, Morone NE, Bost JE, Farrell MH. Prevalence of urinary tract infection in childhood: a meta-analysis. Pediatr Infect Dis J 2008;27:302-8. doi:10.1097/INF.0b013e31815e4122.

3 Hay AD, Whiting P, Butler CC. How best to diagnose urinary tract infection in preschool children in primary care?. BMJ 2011;343:d6316. doi:10.1136/bmj.d6316.

4 Urinary tract infection in children: diagnosis, treatment and long-term management London, England: National Institute for Health and Clinical Excellence; 2007. www.nice.org.uk/nicemedia/ live/11819/36032/36032.pdf accessed May 4, 2012.

5 Coulthard MG, Lambert HJ, Vernon SJ, Hunter EW, Keir MJ, Matthews JN. Does prompt treatment of urinary tract infection in preschool children prevent renal scarring: mixed retrospective and prospective audits. Arch Dis Child 2014;99:342-7. doi:10.1136/ archdischild-2013-304428

6 Shaikh N, Mattoo TK, Keren R, et al. Early antibiotic treatment for pediatric febrile urinary tract infection and renal scarring. JAMA Pediatr 2016:170:848-54 doi:10.1001/jamapediatrics.2016.1181.

7 Roberts KB. Subcommittee on Urinary Tract Infection, Steering Committee on Quality Improvement and Management. Urinary tract infection: clinical practice guideline for the diagnosis and management of the initial UTI in febrile infants and children 2 to 24 months. Pediatrics 2011;128:595-610. doi:10.1542/peds.2011-1330.

8 Mori R, Yonemoto N, Fitzgerald A, Tullus K, Verrier-Jones K, Lakhanpaul $M$. Diagnostic performance of urine dipstick testing in children with suspected UTI: a systematic review of relationship with age and comparison with microscopy. Acta Paediatr 2010:99:581-4.doi:10.1111/i.1651-2227.2009.01644.x

9 Buntsma D, Stock A, Bevan C, Babl FE. Paediatric Research in Emergency Departments International Collaborative (PREDICT). How do clinicians obtain urine samples in young children?Emerg Med Australas 2012;24:118-9. doi:10.1111/j.1742-6723.2011.01518.x.

10 Coutinho K, Stensland K, Akhavan A, Jayadevan R, Stock JA. Pediatrician noncompliance with the American Academy of Pediatrics guidelines for the workup of UTI in infants. Clin Pediatr (Phila) 2014;53:1139-48. doi:10.1177/0009922814536263.

11 Hadjipanayis A, Grossman Z, Del Torso S, et al. Current primary care management of children aged 1-36 months with urinary tract infections in Europe: large scale survey of paediatric practice. Arch Dis Child 2015;100:341-7. doi:10.1136/archdischild-2014306119.

12 Coulthard MG. NICE on childhood UTI: Nasty processes produce nasty guidelines. BMJ 2007;335:463, author reply 463-4. doi:10.1136/ bmi.39325.436667.3A.

13 Tullus K. A review of guidelines for urinary tract infections in children younger than 2 years. Pediatr Ann 2013;42:52-6 doi:10.3928/00904481-20130222-10.

14 Kennedy KM, Glynn LG, Dineen B. A survey of the management of urinary tract infection in children in primary care and comparison with the NICE guidelines. BMC Fam Pract 2010;11:6 doi:10.1186/1471-2296-11-6.

15 Ochoa Sangrador C, Pascual Terrazas A. [Systematic review of the validity of urine cultures collected by sterile perineal bags]. An Pediat (Barc) 2016;84:97-105. doi:10.1016/i.anpedi.2015.04.003.

16 Tosif S, Baker A, Oakley E, Donath S, Babl FE. Contamination rates of different urine collection methods for the diagnosis of urinary tract infections in young children: an observational cohort study. J Paediatr Child Health 2012:48:659-64 doi:10.1111/j.1440-1754.2012.02449.x

17 Herreros ML, Tagarro A, García-Pose A, Sánchez A, Cañete A, Gili P. Accuracy of a new clean-catch technique for diagnosis of urinary tract infection in infants younger than 90 days of age. Paediatr Child Health 2015;20:e30-2.

18 Altuntas N, Tayfur AC, Kocak M, Razi HC, Akkurt S. Midstream clean-catch urine collection in newborns: a randomized controlled study. Eur J Pediatr 2015;174:577-82. doi:10.1007/ s00431-014-2434-z.

19 Alam MT, Coulter JB, Pacheco J, et al. Comparison of urine contamination rates using three different methods of collection: clean-catch, cotton wool pad and urine bag. Ann Trop Paediatr 2005;25:29-34. doi:10.1179/146532805X23326

20 Davies P, Greenwood R, Benger J. Randomised trial of a vibrating bladder stimulator--the time to pee study. Arch Dis Child 2008:93:423-4. doi:10.1136/adc.2007.116160.

21 Kaufman J, Tosif S, Fitzpatrick P, et al. Urine clean catch in the paediatric emergency department: success, time to void and contamination rates. Canadian Pediatric Society Annual Conference. Charlottetown, 2016 
22 Copp HL, Yiee JH, Smith A, Hanley J, Saigal CS. Urologic Diseases in America Project. Use of urine testing in outpatients treated for urinary tract infection Pediatrics 2013:132:437-44 doi:10.1542/ peds.2012-3135.

23 Tran A, Fortier C, Giovannini-Chami L, et al. Evaluation of the bladder stimulation technique to collect midstream urine in infants in a pediatric emergency department. PLoS One 2016;11:e0152598. doi:10.1371/journal.pone.0152598.

24 Whiting P, Westwood M, Bojke L, et al. Clinical effectiveness and cost-effectiveness of tests for the diagnosis and investigation of urinary tract infection in children: a systematic review and economic model. Health Technol Assess 2006;10:iii-iv, xi-xiii, 1-154.

25 Wu HY, de Groat WC. Maternal separation uncouples reflex from spontaneous voiding in rat pups. J Urol 2006;175:1148-51. doi:10.1016/S0022-5347(05)00321-6

26 Tai C, Shen B, Wang J, Chancellor MB, Roppolo JR, de Groat WC. Inhibitory and excitatory perigenital-to-bladder spinal reflexes in the cat. Am J Physiol Renal Physiol 2008;294:F591-602. doi:10.1152/ ajprenal.00443.2007.

27 Fowler Cl, Griffiths D, de Groat WC. The neural control of micturition. Nat Rev Neurosci 2008;9:453-66. doi:10.1038/nrn2401.

28 Kaufman J, Fitzpatrick P, Tosif S, et al. The QuickWee trial: protocol for a randomised controlled trial of gentle suprapubic cutaneous stimulation to hasten non-invasive urine collection from infants. BMJ Open 2016;6:e011357. doi:10.1136/bmjopen-2016-011357.

29 Moher D, Hopewell S, Schulz KF, et al. CONSORT 2010 explanation and elaboration: updated guidelines for reporting parallel group randomised trials. BMJ 2010;340:c869. doi:10.1136/bmj.c869.

30 Kaufman J, Tosif S, Fitzpatrick P, et al. Quick-Wee: a novel non-invasive urine collection method. Emerg Med / 2017;34:63-4. doi:10.1136/ emermed-2016-206000.

31 Herreros Fernández ML, González Merino N, Tagarro García A, et al. A new technique for fast and safe collection of urine in newborns. Arch Dis Child 2013:98:27-9. doi:10.1136/archdischild-2012-301872.

32 Harris PA, Taylor R, Thielke R, Payne J, Gonzalez N, Conde JG. Research electronic data capture (REDCap)--a metadata-driven methodology and workflow process for providing translational research informatics support. J Biomed Inform 2009;42:377-81. doi:10.1016/j.jbi.2008.08.010.
33 Tosif S, Kaufman J, Fitzpatrick P, et al. Urine clean catch in the paediatric emergency department: time to void and contamination rate. Australasian College for Emergency Medicine Annual Scientific Meeting, Melbourne, Australia, 2014.

34 Schulz KF, Altman DG, Moher D. CONSORT Group. CONSORT 2010 statement: updated guidelines for reporting parallel group randomised trials. BM/ 2010:340:c332 doi:10.1136/bmi.c332.

35 Tran VT, Ravaud P. Frugal innovation in medicine for low resource settings. BMC Med 2016;14:102. doi:10.1186/s12916-016-0651-1.

36 Taylor MR, Dillon M, Keane CT. Reduction of mixed growth rates in urine by using a "finger tap" method of collection. BMJ 1986;292:990. doi:10.1136/bmj.292.6526.990.

37 Valleix-Leclerc M, Bahans C, Tahir A, et al. Prospective evaluation of a cutaneous stimulation technique to induce on-demand urination in non-toilet-trained infants. Arch Pediatr 2016;23:815-9. doi:10.1016/ j.arcped.2016.05.004.

38 Labrosse M, Levy A, Autmizguine J, Gravel J. Evaluation of a new strategy for clean-catch urine in infants. Pediatrics 2016;138:e20160573. doi:10.1542/peds.2016-0573.

39 Wu HY. Achieving urinary continence in children. Nat Rev Urol 2010;7:371-7. doi:10.1038/nrurol.2010.78

40 Tullus K, Hooman N, Easty M. Flushing of the vagina and the prepuce-a cause for contaminated urine cultures in children. Pediatr Nephrol 2017:32.107-11. doi:10.1007/s00467-016-3463-9.

41 Ballouhey Q, Fourcade L, Couve-Deacon E, et al. Urine contamination in nontoilet-trained and uncircumcised boys. Urology 2016;95:171-4 doi:10.1016/j.urology.2016.05.056.

42 Vaillancourt S, McGillivray D, Zhang X, Kramer MS. To clean or not to clean: effect on contamination rates in midstream urine collections in toilet-trained children. Pediatrics 2007;119:e1288-93. doi:10.1542/ peds.2006-2392.

43 Tullus K. Difficulties in diagnosing urinary tract infections in small children. Pediatr Nephrol 2011;26:1923-6. doi:10.1007/ s00467-011-1966-y.

44 O'Brien K, Edwards A, Hood K, Butler CC. Prevalence of urinary tract infection in acutely unwell children in general practice: a prospective study with systematic urine sampling. Br I Gen Pract 2013;63:e15664. doi:10.3399/bjgp13X663127. 